I. II.

$\begin{array}{lrr}\text { Kohlenstoff } & 78,11 & 78,49 \\ \text { Wasserstoff } & 12,99 & 13,21 \\ \text { Sauerstoff } & 8,90 & 8,30 .\end{array}$

Diese Zahlen entsprechen sehr gut der Formel

man hat nämlich :

$$
\begin{array}{lll}
\mathrm{C}_{72} & \mathrm{H}_{144} & \mathrm{O}_{6}
\end{array}
$$

$$
\begin{array}{lr}
\mathrm{C}_{72}=5400,0 & 78,26 \\
\mathrm{H}_{144}=900,0 & 13,04 \\
\mathrm{O}_{\mathfrak{6}}=\frac{600,0}{6900,0} & \frac{8,69}{99,99}
\end{array}
$$

Bei der Behandlung des chinesischen Wachses mit Salpetersäure scheinen sich dieselben Producte zu bilden als die sind, welche man erlält, wenn man diese Säure auf das Bienenwachs einwirken lässt; unter anderen Producten bildet sich eine nüchtige Säure, welche die Hauptcharaktere der Buttersäure besilzt.

\title{
IV.
}

Ueber die Vegetation, vom chemischen Gesichtspuncte betrachtet.

Von

7. C. Calvert and I. Fernand.

(Compt. rend. XVII. No. 18. 30. Oct. 18+3; Auszug von d. Vff.)

Wir haben uns vorgenommen, durch die chemische Analyse die Veränderungen zn untersuchen, welche die Zusammensetzung der in den Pflanzen selbst enthaltenen Luft exleidet, sowohl nach den Organen, als anch nach den Umständen, welchen die hauptsächlichsten Erscheinungen der Vegetation ausgesetzt sind.

I. Unsere Versuche, welche die Zersetzung der Kohlensäure durch die Planzen unter dem Einflusse des Sonnenlichtes betreffen, haben wir unter den günstigsten Bedingungen angestellt, indem, während wir die in gewissen Theilen der Pflanzen ent- 
haltene Laff untersuchten, die Planze selbst lebend in der Erde blieb *).

II. Ferner unternahmen wir die chemische Untersuchung der in den Schoten von Colutea arborescens enthaltenen Luft.

Diese Schoten, welche uns bei unseren Untersuchungen als Typus für die Früchle dienten, da sie von der äussern Luft nur in sehr beschränktem Maasse durchdringbar sind, wovon wir uns vorher überzeugten, haben uns gestattet, den Modificationen zu folgen, welche die Kohlensïure, die sie enthalten, je nach den Umständen erleidet, in der Dunkelheit, beim Tages- oder beim Sonnenlichte, nach den verschiedenen Stunden des Tages und zufolge der verschiedenen Perioden der Entwickelung, deren wir hanptsüchlich drei angenommen haben: junge Schoten, intermediüre Schoten und alte Schoten. Diese Früchte wurden unmittelbar nach ihrer Einsammlung unter Quecksilber in zu diesem Zwecke eingerichteten Glocken geöffnet und die Feuchtigkeit des erhaltenen Gases von der Kohlensüure mit Hülfe von Schwefelsäure mittelst des kleinen, in unserer Abhindlung beschriebenen Apparates entfernt. Nach dieser ersten Operation wurde das getrocknete Gas in graduirte Glocken gebracht, in welchen das kaustische Kali nach 24 Stunden die Absorption der Kohlensäure anzeigte.

Wir haben Perner, um die. Menge des Sauerstoffes zu erfahren, uns des Eudiometers mit Wasserstoffgas bedient, wobei wir alle Vorsichtsmaassregeln anwandten, welche diese Methode der Analyse erfordert. In allen Fällen, sei es bei der Bestimmung der Kohlensäure, sei es bei der Messung des Sauerstoffes, haben wir immer die in der Berechnung zufolge der Variationen der Temperatur und des Druckes nothwendigen Correctionen rorgenommen.

Wir begnügen uns hier, die vergleichende Tabelle zu geben, der mittleren Mengen der in den Schoten von Colutea arborescens, je nach dem Zustande des Himmels und der Zeit unserer Versuche, enthaltenen. Kohlensüure und Sauerstoffes.

*) Vir baben auf diese Art alle die Phasen bei der Assimilation des Kuhlenstoffes verfulgen können, dadurch, dass wir die Wirkung der Pflanze auf eingeschlossene Luft untersuchten, eine Bestimmung, die uns vernïnftiger und zweckmässiger zu sein scheint als die Analyse der nmgebenden Luft. 
Calvert a. Fernand: Ueber d. Vegetation.

Intermediäre Schoten.

\begin{tabular}{r|l|c|c|c}
\hline $\begin{array}{c}\text { Stunde } \\
\text { des } \\
\text { Ver- } \\
\text { suches. }\end{array}$ & \multicolumn{1}{|c|}{$\begin{array}{c}\text { Zustand } \\
\text { des Himmels. }\end{array}$} & $\begin{array}{c}\text { Sauerstoff } \\
\text { in } 100 \\
\text { dem Volumen } \\
\text { nach. }\end{array}$ & $\begin{array}{c}\text { Kohlensäure } \\
\text { in 100 } \\
\text { dem Volumen } \\
\text { nach. }\end{array}$ & $\begin{array}{c}\text { Kohlensäure } \\
\text { u. Sauerstoff } \\
\text { zusammen. }\end{array}$ \\
\hline 11 & Nacht & 20,496 & 2,746 & $\mathbf{2 3 , 2 4 2}$ \\
7 & Morgen, bedeckt & 20,673 & 2,618 & 23,291 \\
12 & Mittag, bedeckt & 20,908 & 2,429 & 23,337 \\
4 & Nachmittag, bedeckt & 20,901 & 2,432 & 23,383 \\
7 & Morgen, Sonne & 21,086 & 1,903 & 22,989 \\
12 & Mittag, Sonne & 21,293 & 1,419 & 22,712 \\
4 & Nachmittag, Sonne & 21,176 & 1,438 & 22,614 \\
& & & &
\end{tabular}

Junge Schoten.

\begin{tabular}{r|l|l|l|l}
11 & Nacht & 20,583 & $\mathbf{2 , 6 3 9}$ & $\mathbf{2 3 , 2 2 2}$ \\
7 & Morgen, bedeckt & 20,626 & $\mathbf{2 , 6 0 5}$ & 23,231 \\
12 & Mittag, bedeckt & 20,766 & $\mathbf{2 , 4 4 6}$ & 23,012 \\
4 & Nachmittag, bedeckt & $20,7+3$ & 2,475 & 23,218 \\
7 & Morgen, Sonne & 20,844 & 1,934 & 22,778 \\
12 & Mittag, Sonne & 21,032 & $\mathbf{1 , 7 6 2}$ & 22,794 \\
4 & Nachmittag, Sonne & 21,246 & 2,0983 & 23,339 \\
\hline
\end{tabular}

\section{Alte Schoten.}

\begin{tabular}{r|l|l|l|l}
11 & Nacht & 19,297 & 2,942 & 22,239 \\
7 & Morgen, bedeckt & 20,166 & $\mathbf{2 , 6 0 9}$ & $\mathbf{2 2 , 7 7 5}$ \\
12 & Mittag, bedeckt & 20,626 & $\mathbf{2 , 4 6 1}$ & 23,087 \\
4 & Nachmittag, bedeckt & 20,595 & $\mathbf{2 , 4 7 5}$ & $\mathbf{2 3 , 0 7 0}$ \\
7 & Morgen, Sonne & 21,139 & $\mathbf{2 , 3 1 6}$ & $\mathbf{2 3 , 4 5 5}$ \\
12 & Mittag, Sonne & $\mathbf{2 1 , 2 4 6}$ & $\mathbf{2 , 1 0 6}$ & $\mathbf{2 3 , 3 4 2}$ \\
4 & Nachmittag, Sonne & 20,676 & $\mathbf{2 , 1 0 7}$ & $\mathbf{2 2 , 7 8 3}$ \\
\hline
\end{tabular}

\section{Betrachtungen über diese Tabelle.}

1) Diese numerischen Resultate zeigen, dass die Luft der Schoten weit reicher an Kohlensäure ist als die atmosphärische Luft.

2) Sie zeigen auf eine schlagende Weise, dass die Menge der Kohlensäure bei Nacht beträchtlicher ist als am Tage; und wenn man die beiden äussersten Beispiele nimmt, dasjenige um 11 Uhr Nachts $(2 ; 746)$ und dasjenige in dem Augenblicke, in welchem das Licht das Maximum seiner Intensität zeigt $(1,419)$, so. sieht man, dass das Verhältniss in dem einen Falle zweimal grösser ist als in dem andern.

Journ. f. prakt. Chemie. XXXI. 1. 
3) Diese Tabelle, wenn man von den in der Nacht angestellten Versuchen ausgeht, gestattet ferner, die progressive $\mathbf{A b}$ nahme der Kohlensäure bis zu dem Puncte zu verfolgen, wo dieselbe aufzuhören scheint. Man sieht also, dass die zersetzende Kraft des Lichtes sich mit dessen Intensität und der Dauer seiner Wirkung vermehrt, sei es nun, dass man die verschiedenen Stunden eines und desselben schönen oder unfreundlichen Tages mit einander vergleicht, oder -dass man die bei einem ganz bedeckten Himmel und die bei starkem Sonnenscheine erhaltenen Resultate betrachtet.

4) Man bemerkt ferner, dass hinsichtlich des Alters der Schoten die Reduction der Kohlensäure mit der Kraft der Vegelation in Verhältniss steht.

5) Als Beweis für die sehr geringe Durchdringbarkeit der Fruchthülsen des Blasenbaumes sieht man in der Columne des Sauerstoffes, dass die Verhältnisse dieses Gases sich in der Frucht in dem Maasse vermehren, als die Kohlensäure sich in derselben zersetzt; die Beziehungen, welche zwischen dem Verschwinden der Kohlensüure und der Vermehrung des Sauerstoffes bestehen, sind genau von der Art, dass dieser hinzugekommene Sauerstoff betrachtet werden kann als herrührend von der Säure, welche, indem sie sich zersetzt, ihren Kohlenstoff an die Pflanze abgiebt.

6) Wir bemerken ferner 1) dass, wenn man den Sauerstoff zur Kohlensäure hinzuaddirt, man als Mittelzahl 23 erhält; 2) dass die Kohlensäure immer Stickstoff und zuweilen ein wenig Sauerstoff austreibt; aber das Letztere ist nur der Fall, wenn die Quantität der Kohlensäure gross ist, wie sie das erste Beispiel einer jeden Reihe angiebt.

Die Versuche von Sennebier, de Sausure und diejenigen ron Dumas, Boussingault, Liebig haben die Fixation des Kohlenstoffes durch die Pflanzen bewiesen: aber man wird uns vielleicht Dank wissen, durch diese Resultate die Art und Weise der Wirkung kennen gelehrt zu haben, welche das Licht auf die Reduction ausübt, welche mit der Morgendämmerung anfängt und während des Tages beim gewöhnlichen Tageslichte fortgesetzt wird; dieses Resultat stimmt nicht mit dem überein, was man hinsichtlich der Fixation des Kohlenstof- 
fes annahm, da man glaubte, dass diese allein in dem Falle stattfinde, wenn die Pflanze von den Strahlen der Sonne direct getroffen würde.

III. Die dritte Abtheilung unserer Abhandlung umfasst die chemische Untersuchung der Luft in den Räumen einer gewissen Anzall von hohlen Stengeln, aus denen wir unmittelbar bei ihrer Einsammlung die Gase unter mit Queeksilber angefüllten Glocken haben aufsteigen lassen. Bei den bei dieser Arbeit nothwendigen Manipulationen sind mit Sorgfalt alle die Umstände vermie. den worden, welche eine Mischung der Luft in den Stengeln mit der äussern Luft verursachen könnten.

Die Gase, welche erhalten und wie diejenigen aus den Schoten durch Schwefelsäure getrocknet wurden, haben uns bei der Behandlung mit kaustischem Kali und auf eudiometrischem Wege untersucht, folgende Resultate gegeben:

Tabelle uiber die Quantitäten der Kohlensäure, dem Volumen nach.

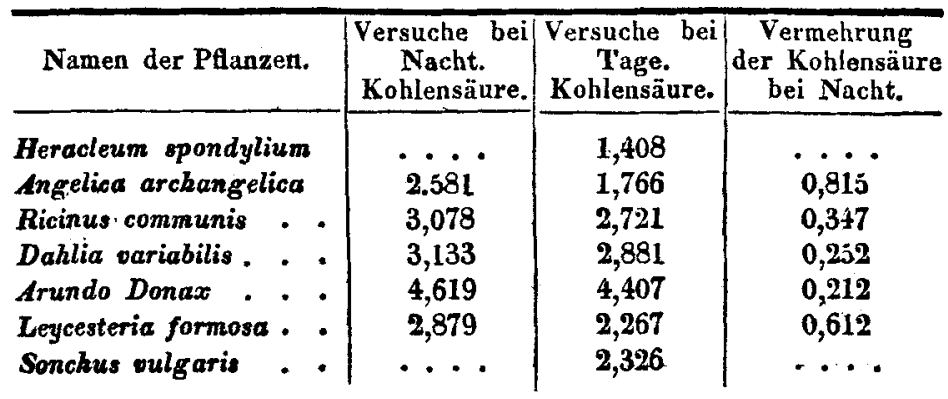

Tabelle über die Menge des Sauerstoffes, dem Volumen nach.

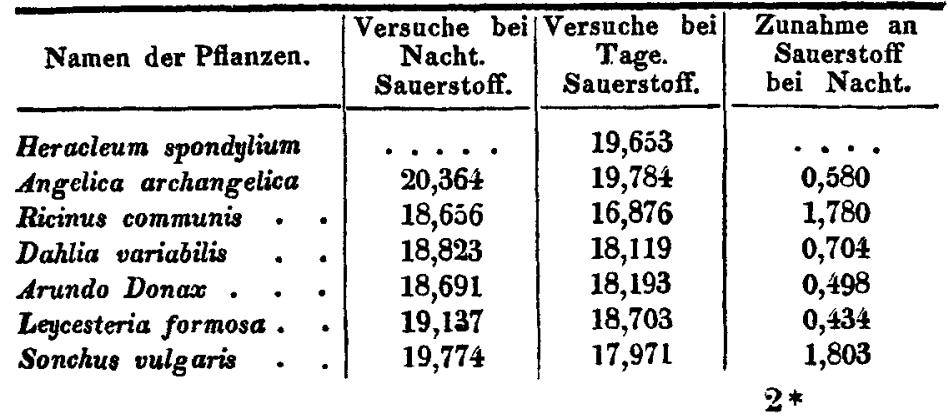


1) Es folgt aus diesen Tabellen, dass die in den Stengeln enthaltene Luft eine eigenthümliche, von derjenigen der atmosphärischen Luft sehr verschiedene Zusammensetzung hat, da sie, abgesehen vom Sauerstoff, die grosse Menge Kohlensäure enthält, welche Menge sich mit der Kraft der Vegetation vermehrt.

2) Es folgt ferner aus dem Angeführten, dass die Henge der Kohlensäure grüsser bei Nacht als bei Tage ist, aber dass die Differenz nicht so bedeutend ist, als es mit den Hülsenfrüchten der Fall war. Diese zweite Thatsache lässt sich, nach unserer Meinung, durch den Unstand erklären, dass nämlich der ganze Stengel des caudex descendens, ascendens und die Wurzeln an der Absorption Theil haben, während Verminderung der Kohlensäure nur in dem Theile des caudex ascendens stattfindet, dessen Oberfläche der zersetzenden Einwirkung des Lichtes ausgesetzt ist.

3) Wir können noch bemerken, dass in den Stengeln der Sauerstoff sich des Nachts zugleich mit der Kohlensäure vermehrt, wovon wir das Gegentheil bei den Schoten, welche wir untersuchten, gesehen haben.

IV. Der wichtige Einfluss des Ammoniaks in der Vegetation ist in der letzten Zeit durch die gelehrten Untersuchungen der Herren Dumas, Boussingault, Liebig ausser Zweifel gesetzt worden; da aber eine Stelle des, ,'Essai de Statique chimique des êtres organisés" yon $\mathrm{D}$ u mas bei uns einigen $\mathrm{Zweifel}$ über diesen Gegenstand übrig liess, so haben wir geglaubt, dass es für die Wissenschaft von Interesse sein würde, wenn wir untersuchten, ob das Ammoniak der Luft die Gegenwart des in den Pflanzen gebundenen Stickstoffes bewirke, und wir glauben diese Thatsache auf eine deutliche Weise nachgewiesen zu haben, indem wir das Ammoniak im gasförmigen Zustande in der Luft entdeckten, welche die Pflanzen einschliessen. 
sestimmung des Ammoniaks im Zustande con Platinsalmiak.

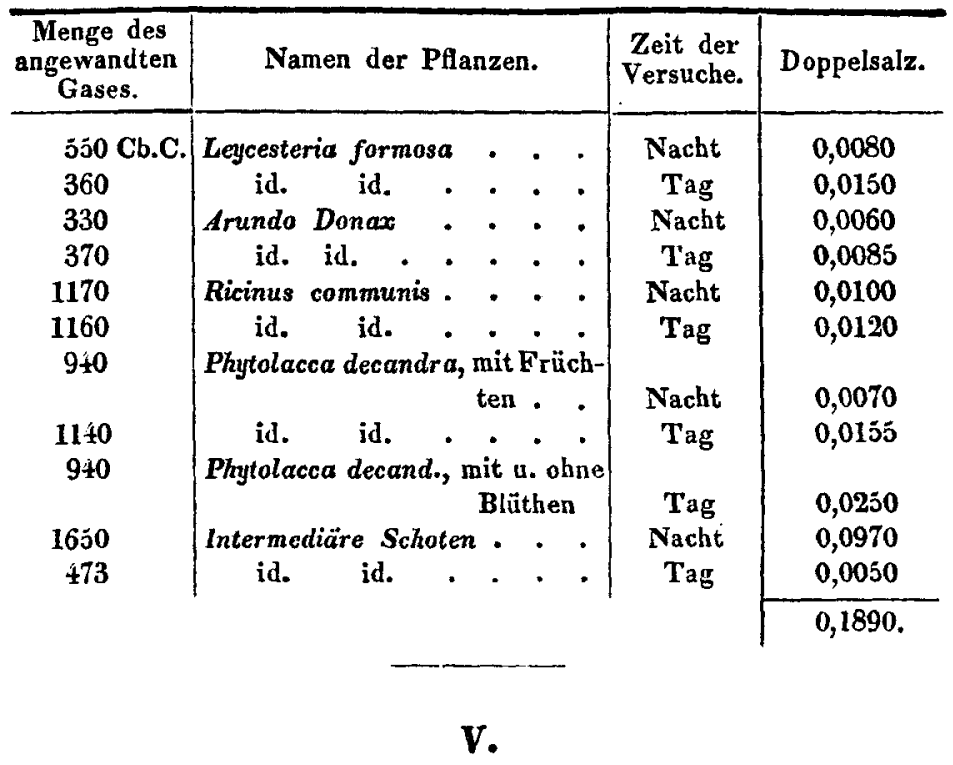

Ueber die Zersetzung des Kohlensäuregases und der kohlensauren Alkalien durch das Sonnenlicht.

Von

\section{John W. Iraper.}

(The Lund., Edinb. and Dubl. phil. Mag., September 1843. p. 161.)

Es ist schon seit vielen Jahren bekannt, dass die grünen Theile der Pflanzen unter dem Einflusse des Sonnenlichtes das Vermögen besitzen, Kohlensäure zu zersetzen und ihren Sauerstoff frei zu machen. Es ist merkwürdig, dass diess, was eine Fundamentalthatsache in der Pflanzenphysiologie ist, noch nicht genau untersucht worden ist. Die in den Büchern darüber vorkommenden Angaben sind oft durchaus nicht richtig. Es wird zuweilen gesagt, dass sich reines Sauerstoffgas entwickelt, dass die Zersetzung durch die sogenannten chemischen Strahlen bewirkt wird. Diese und eine Menge von anderen dergleichen Irrthümern sind im Umlanfe. So weit sich meine Lectüre erstreckt, hat noch Niemand eine Erklärung der Erscheinung vermittelst des Prisma's versucht, welches doch der einzige Weg ist, auf dem dieselbe richtig erörtert werden kann. 